\title{
Coproducts of De Morgan algebras
}

\section{William H. Cornish and Peter R. Fowler}

The dual of the category of De Morgan algebras is described in terms of compact totally ordered-disconnected ordered topological spaces which possess an involutorial homeomorphism that is also a dual order-isomorphism. This description is used to study the coproduct of an arbitrary collection of De Morgan algebras and also to represent the coproduct of two De Morgan algebras in terms of the continuous order-preserving functions from the Priestley space of one algebra to the other algebra, endowed with the discrete topology. In addition, it is proved that the coproduct of a family of Kleene algebras in the category of De Morgan algebras is the same as the coproduct in the subcategory of Kleene algebras if and only if at most one of the algebras is not boolean.

\section{Preliminaries}

A De Morgan algebra $(M ; \vee, \wedge, \sim, 0,1)$ is an algebra of type $\langle 2,2,1,0,0\rangle$ such that $(M ; v, \wedge, 0,1)$ is a distributive lattice with largest element $I$ and smallest element 0 and $\sim$ is an involutorial dual order-(lattice-)isomorphism; that is, the equations $\sim x=x$, $\sim(x \vee y)=\sim x \wedge \sim y, \sim(x \wedge y)=\sim x \vee \sim y, \sim 0=1$, and $\sim 1=0$ are identically satisfied. A Kleene algebra is a De Morgan algebra which satisfies the identity associated with the inequality $x \wedge \sim x \leq y \vee \sim y$. In this paper, a boolean algebra will be regarded as a De Morgan algebra satisfying the identity $x \wedge \sim x=0$; of course, a boolean algebra is a Kleene algebra. The chain

$$
\left\{0, x_{1}, \ldots, x_{n-2}, 1: 0=x_{0}<x_{1}<\ldots<x_{n-2}<x_{n-1}=1\right\}
$$

Received 13 August 1976. 
with $n$ elements possesses a unique involutorial dual order-isomorphism such that it becomes a kleene (De Morgan) algebra; for $i=0, \ldots, n-1$, $x_{i}=x_{n-i-1}$, and so if $n$ is odd the element $x_{(n-1) / 2}$ is fixed under the involution. We will denote the De Morgan algebra associated with the chain possessing $n$ elements by $n$. If a De Morgan algebra $M$ is not boolean then there exists $x \in M$ such that $0<x \wedge \sim x \leq x \vee \sim x<1$ and so a De Morgan algebra is not boolean if and only if it possesses a subalgebra isomorphic to either 3 or 4 . The category whose objects are members of the variety of De Morgan algebras and whose morphisms are the associated algebra-homomorphisms is denoted by $M$; the subcategories which correspond to the subvarieties of Kleene algebras and boolean algebras are denoted by $K$ and $B$, respectively. We will use $D$ to denote the category whose objects are distributive lattices $(L ; \vee, \wedge, 0,1)$ with 0 and 1 , and whose morphisms are the associated algebra-homomorphisms. For reasons of emphasis, it will sometimes be convenient to describe the homomorphisms, congruences, and subalgebras associated with De Morgan algebras as $\sim$-homomorphisms, -congruences, and $\sim$ subalgebras, respectively. Let $X$ be any one of the categories $M, K, B$ or $D$. Then the coproduct in the category $X$ of a set $\left\{Y_{i}\right\}$ of objects in $X$ is the same as their free product in the variety $X$ ( $[1$, Theorem 7 , p. 34]) and is denoted by $\perp_{X}{ }^{Y} ; Y \perp_{X}{ }^{Z}$ is used to denote the coproduct of two objects $Y, Z$ of $X$. Coproducts of an arbitrary set of objects in $D$ are described, in detail, in Grätzer [9, Section 12, pp. 128-137] and Balbes and Dwinger [1, Chapter 7, pp. 132-150], while coproducts in $M$ are described in [1, Section 11.4, pp. 216-218]. Representations of the coproduct of two objects $L_{1}, L_{2}$ of $D$ in terms of all the continuous monotone (order-preserving) functions from the Priestley space of $L_{1}$ into $L_{2}$, endowed with the discrete topology, have been given independently by the first author [7] and Davey [8]. One of the aims of this paper is to extend the representations of [7], [8] to $M$.

Let $C(X)$ be the family of all compact-open subsets of a topological space $X$. Then the topological space $X$ is called a spectral space if it satisfies each of the following properties:

(SI) $X$ is a compact $T_{0}$-space; 
(s2) $\mathcal{C}(X)$ is a ring of subsets of $X$ and a base for the open sets;

(S3) if $F$ is a closed set in $X$ and $C_{1}$ is any subfamily of $C(X)$ such that $\cap F_{I} \cap F \neq \varnothing$ for any finite subfamily $F_{I}$ of $\mathcal{C}_{1}$ then $\cap \mathcal{C}_{1} \cap F \neq \emptyset$.

A function between topological spaces is called strongly continuous if it is continuous and the inverse image of each compact-open subset is itself compact-open. The category whose objects are spectral spaces and whose morphisms are strongly continuous functions is denoted by Spec.

An ordered topological space $(X ; \leq, \tau)$ is a partially ordered set $(X ; \leq)$ and a topological space $(X ; \tau)$; when there is no possibility of confusion, we will denote an ordered topological space $(X ; \leq, \tau)$ by means of the underlying set $X$. An ordered topological space $X$ is totally order-disconnected if, whenever $x \neq y(x, y \in X)$, there exist a clopen increasing subset $U$ and a clopen decreasing subset $V$ such that $U \cap V=\varnothing$ and $x \in U$, while $y \in V$ - a subset $U$ of a partially ordered set $X$ is increasing (decreasing) if $x \leq y$ and $x \in U(y \in U)$ imply $y \in U(x \in U)$. The set $D(X)$ of all clopen increasing subsets of an ordered topological space $X$ is a bounded distributive lattice with respect to set-union and set-intersection. In a compact totally orderdisconnected ordered topological space $X, D(X) \cup\{X \backslash U: U \in D(X)\}$ is a sub-base for the open sets ( $c f$. [6, Section 1]). The category whose objects are compact totally order-disconnected ordered topological spaces and whose morphisms are continuous monotone functions is denoted by Todc. In [6, Theorem 2.3] it is shown that Spec and Todc are isomorphic categories. It is well known that Spec is the dual of the category $D$ (cf. [9, Section 11, pp. 117-125], [1, Chapter 4, pp. 75-84]) and hence, as noted in [6], Tode is also the dual of $D$.

Let $L$ be an object of $D$ and let $\Sigma(L)$ be the set of all prime ideals of $L$. When $\Sigma(L)$ is endowed with the so-called hull-kernel or Stone topology, whose basic open sets are those sets of the form $\{P \in \Sigma(L): a \notin P\}$ for some $a \in L$, it is a spectral space and the map $a \mapsto\{P \in \Sigma(L): a \mid P\}$ is an isomorphism of $L$ onto $\mathcal{C}(\Sigma(L))$. The space $\Sigma(L)$ is called the Stone space of $L$. Order $\Sigma(L)$ with that 
partial order which is the converse of set-inclusion and endow $\Sigma(L)$ with the topology whose sub-basic open sets are of the form $\{P \in \Sigma(L): a \notin P\}$ or $\{P \in \Sigma(L): a \in P\}$, where $a \in L$. That is,

$$
C(\Sigma(L)) \cup\{X \backslash V: V \in C(\Sigma(L))\}
$$

is a sub-base for the new topology. Then $\Sigma(L)$ becomes an object in Tode (cf. [6], [5, Corollary 1.5]). This ordered space is called the Priestley space of $L$ and is denoted by $\operatorname{Pr}(L) ; \mathcal{C}(\Sigma(L))$ can be identified with $D(\operatorname{Pr}(L))$ and the map $a \mapsto\{P \in \operatorname{Pr}(L): a \mid P\}$ is an isomorphism of $L$ onto $\operatorname{Pr}(L)$.

\section{Coproducts}

Let $P$ be a prime ideal in a De Morgan algebra $M$ and let $g(P)=M \sim P$, where for any subset $A$ of $M, \sim A=\{\sim a: a \in A\}$. Then $g(P)$ is also a prime ideal and the map $P \mapsto g(P)$ is an involution on the Stone space $\Sigma(M)$. Since, for any $a \in M$,

$$
\{P \in \Sigma(M): \sim a \notin P\}=\Sigma(M) \backslash g(\{P \in \Sigma(M): a \notin P\}),
$$

we see that for each $V \in \mathcal{C}(\Sigma(M)), \Sigma(M) \backslash g(V) \in \mathcal{C}(\Sigma(M))$. In addition, if we define $\sim V$ to be $\Sigma(M) \backslash g(V)$ for any $V \in C(\Sigma(M))$ then $c(\Sigma(M))$ becomes a De Morgan algebra and the map $a \mapsto\{P \in \Sigma(M): a \notin P\}$ is a r-isomorphism of $M$ onto $\mathcal{C}(\Sigma(M))$; this was first proved by BiaxynickiBirula and Rasiowa [3]. Let g-Spec be the category whose objects are spectral spaces $X$ possessing an involution $g$ such that $X \backslash g(V) \in C(X)$ for each $V \in \mathcal{C}(X)$ and whose morphisms are strongly continuous functions $f: x_{1} \rightarrow x_{2}\left(\left(x_{1}, g_{1}\right),\left(x_{2}, g_{2}\right)\right.$ objects in g-Spec $)$ such that $g_{2} \circ f=f \circ g_{1}$. Then Petrescu has extended the duality between $D$ and Spec to a duality between $M$ and g-Spec.

LEMMA 2.1 (Petrescu [12, Corollary 2.5]). The category g-Spec is isomorphic to the dual of $M$.

Let $X$ be a spectral space and $f$ be a morphism in Spec. Then the relation $x \leq y \quad(x, y \in X)$ if and only if $x$ is in the closure of $\{y\}$, is a partial order on $X$, and $\Omega(X)=(X ; \leq, \tau)$, where $\tau$ is the topology whose sub-basic open sets are the members of $C(X) \cup\{X \backslash V: V \in \mathcal{C}(X)\}$, is an object in Tode. Let $\Omega(f)=f$. Then 
$\Omega(f)$ is a morphism in Todc. On the other hand, if $Y$ is an object in Todc and $\Psi(Y)=(Y ; \sigma)$ is the set $Y$ endowed with the topology $\sigma$ whose basic open sets are the members of $D(Y)$ then $\Psi(Y)$ is an object in Spec. If $h$ is a morphism in Todc and $\Psi(h)=h$ then $\Psi(h)$ is a morphism in Spec and we have

LEMMA 2.2 ([6, Theorem 2.3]). $\Omega$ and $\Psi$ are mutually inverse isomorphisms between the categories Spec and Todc.

Let $\mathbf{g}$-Todc be the category whose objects are compact totally orderdisconnected ordered topological spaces $X$ which possess an involution $g$, which is both a homeomorphism and a dual order-isomorphism, and whose morphisms are functions $f: X_{1} \rightarrow X_{2} \quad\left(\left(x_{1}, g_{1}\right),\left(x_{2}, g_{2}\right)\right.$ objects in g-Tode) such that $g_{2} \circ f=f \circ g_{1}$.

THEOREM 2.3. The category g-Todc is isomorphic to the dual of the category $M$.

Proof. If $(X, g)$ is an object in g-Spec then both $g(V)$ and $X \backslash g(V)=g(X \backslash V)$ are clopen in $\Omega(X)$ for any $V$ in $C(X)$. Because the topology $\tau$ on $\Omega(X)$ has $\mathcal{C}(X) \cup\{X \backslash V: V \in \mathcal{C}(X)\}$ as a sub-base we see that $g$ is an open mapping on $\Omega(X)$. But $g^{2}=1_{X}$, and so $g$ is a homeomorphism. In addition, if $x, y \in X$ and $x \leq y$, then $x$ is in all basic closed sets which contain $y$. Thus $g(y) \leq g(x)$, as otherwise $g(y) \neq g(x)$ and so there is $W \in \mathcal{C}(X)$ such that $g(y) \in W$ and $g(x) \in X \backslash W$ and so $y \in X \backslash V, V=X \backslash g(W) \in \mathcal{C}(X)$, and yet $x \notin X \backslash V$. Since $g^{2}=I_{X}$ it follows that $g$ is a dual order-isomoxphism. The rest of the theorem follows immediately from Lemmas 2.1 and 2.2 , since $C(X)=D(\Omega(X))$.

If $(x, g)$ is an object in g-Spec then the topology $\tau$ which converts $X$ to $\Omega(X)$ is precisely the coarsest topology on $X$ for which $g$ is continuous or even a homeomorphism. Thus, we see the advantage of using g-Todc as a representation of the dual of $M$. Indeed, we now give a simple proof of a theorem due to Berman and Dwinger (see [1, Theorem 2, p. 216]).

THEOREM 2.4. The product of any set $\left\{\left(x_{i}, g_{i}\right)\right\}$ of objects in 
g-Tode is $\left(T x_{i}, \Gamma g_{i}\right)$, where $T x_{i}$ is the cartesian product of the spaces $X_{i}$ endowed with the direct product order, and $T T g_{i}$ is the product of the functions $g_{i}$. Hence, for any set $\left\{N_{i}\right\}$ of De Morgan azgebras, $\perp_{M} N_{i}$ and $\perp_{D} N_{i}$ are isomorphic Zattices.

Proof. The theorem follows immediately from duality and the fact that $\prod_{g_{i}}: \prod_{x_{i}} \rightarrow \prod x_{i}$ is continuous if and only if each $g_{i}$ is continuous.

COROLLARY 2.5. Let $\left\{M_{i}: i \in I\right\}$ be a set of De Morgan algebras and $J$ be a non-empty subset of $I$ such that for each $j \in J, N_{j}$ is a r-subalgebra of $M_{j}$. Then, provided that each $M_{i}$ is regarded as a $\sim$-subargebra of $\frac{1}{i \in I} M_{i}^{M}$, the r-subalgebra of $\frac{1}{i \in I} M_{i}$. generated by $\bigcup_{j \in J} N_{j}$ is isomorphic to $\frac{1}{j \in J} M_{j}^{N}$.

Proof. Because of Grätzer [9, Theorem 5, p. 131; cf. Corollary 6, p. 132] the sublattice of $\frac{1}{i \in I} D_{i} M_{i}$ generated by $\underset{j \in J}{U} N_{j}$ is isomorphic to $\frac{1}{j \in J} D^{N}$. In view of Theorem 2.4, this sublattice is a De Morgan algebra and, of course, the restrictions of the $\sim$-operations of $\frac{1}{i \in I} M_{i}^{M}$ and the sublattice generated by $\underset{j \in J}{U} N_{j}$ coincide on each $N_{j}$. Hence, the sub lattice generated by $\underset{j \in J}{U} N_{j}$ is a rsubalgebra of $\frac{1}{i \in I} M_{i}^{M}$, and the result follows.

COROLLARY 2.6. Let $\left\{B_{i}\right\}$ be a set of boolean algebras. Then, $\perp_{B}{ }^{B}, \perp L_{K}{ }_{i}, \perp_{M}{ }_{i}$, and $\perp_{D_{i}}$, are isomorphic lattices.

Proof. Due to Theorem 2.4 and $\left[1\right.$, Theorem 2, p. 133], $\perp_{M}{ }_{i}$ is a boolean algebra.

Let $X$ be an ordered topological space and $L$ be a bounded distributive lattice endowed with the discrete topology. Then $C_{m}(X, L)$ denotes the set of all continuous monotone functions mapping $X$ into $L$, 
and $\bar{C}_{m}(X, L)$ is the set of all members of $C_{m}(X, L)$ whose range is finite. Under pointwise operations $\bar{C}_{m}(X, L)$ is a bounded distributive lattice and in [7, Theorem 2.1] it is shown that $\bar{C}_{m}(X, L)$ is isomorphic to $D(X) \perp D^{L}$. Hence, if $A$ is another bounded distributive lattice and $X=\operatorname{Pr}(A), \bar{C}_{m}(X, L)=C_{m}(X, L)$ and $C_{m}(X, L)$ is isomorphic to $A \perp D^{L}$ (cf. [7, Corollary 2.3] and Davey [8]). For $y \in L$, let $c(y)$ be the constant function on $X$ such that $c(y)(x)=y$ for all $x \in X$; the map $y \mapsto c(y)$ is a lattice-embedding of $L$ into $\bar{c}_{m}(X, L)$. For $V \in D(X)$, let $X_{V}$ be the characteristic function of $V$; that is, $\chi_{V}(x)=1$ if $x \in V$ and $\chi_{V}(x)=0$ if $x \in X \backslash V$; the map $V \mapsto \chi_{V}$ is a latticeembedding of $D(X)$ into $\bar{C}_{m}(X, L)$.

Now suppose $(X, g)$ is an ordered topological space with an involutorial homeomorphism $g$ which is also a dual order-isomorphism. If $V \in D(X)$ and we define $\sim V=X \backslash g(V)$ then we see that $D(X)$ becomes a De Morgan algebra. Thus, if $L$ is also a De Morgan algebra, Theorem 2.4 implies that $\bar{C}_{m}(X, L)$ is a De Morgan algebra which is isomorphic to $D(X) \perp_{M} L$. The purpose of the next result is to give a formula for the involution on $\bar{C}_{m}(X, L)$.

THEOREM 2.7. Let $(X, g)$ be an ordered topological space $X$ with an involutorial homeomorphism $g$ which is also a dual order-isomorphism. Let $L$ be a De Morgan algebra. Then $\bar{C}_{m}(X, L)$ is a De Morgan algebra, where, for each $f \in \bar{c}_{m}(X, L), \sim f$ is given by $(\sim f)(x)=\sim(f(g(x)))$ for each $x \in X$, and $\bar{C}_{m}(X, L)$ is isomorphic to $D(X) \perp_{M} L$.

Proof. Let $f \in \bar{C}_{m}(X, L)$. Then $\sim f$, as defined above, is equal to the product $\sim \circ f \circ g$. Hence $\sim f$ has finite range, is monotone as it is the composition of a monotone function together with two antitone functions, and is continuous since $f$ and $g$ are continuous and the roperation on $L$ is continuous because $L$ has the discrete topology. Since $\sim f=\sim \circ(\sim \circ f \circ g) \circ g=\sim^{2} \circ f \circ g^{2}=I_{L} \circ f \circ I_{X}$ and because 
it is easy to see that $\sim=1$ and $f_{1} \leq f_{2}\left(f_{1}, f_{2} \in \bar{C}_{m}(X, L)\right)$ implies $\sim f_{2} \leq \sim f_{1}, \bar{C}_{m}(X, L)$ is a De Morgan algebra with the roperation, as defined above. In order to prove that $\bar{C}_{m}(X, L)$ is isomorphic to $D(X) \perp \perp_{M} L$, it is sufficient, in the light of our remarks preceding this theorem, to check that the maps $y \mapsto c(y)$ and $V \mapsto x_{V}$ are uhomomorphisms of $L$ and $D(X)$, respectively, into $\bar{C}_{m}(X, L)$. But it is not hard to verify that $c(\sim y)=\sim c(y)$ for any $y \in L$ and $\sim \chi_{V}=X_{X \backslash g}(V)=\chi_{\sim V}$ for any $V \in D(X)$, and so the theorem follows.

The representation of $A \perp D^{L}$ ( $A, L$ objects in $\left.D\right)$ by means of $C_{m}(\operatorname{Pr}(A), L)$ can be simplified when $A$ is a chain with $n$ elements (see, for example, [7, Example 2.13]). We now note the corresponding result for $M$, when $A=n$ and $L$ is a De Morgan algebra.

COROLLARY 2.8. Let $L$ be a De Morgan azgebra. Then $n \perp_{M} L$ is isomorphic to the subalgebra of the $(n-1)$-fold direct power of $L$ consisting of all (n-1)-tuples $\left(y_{0}, y_{1}, \ldots, y_{n-2}\right)$ such that $y_{0} \geq y_{1} \geq \ldots \geq y_{n-2}$, and $\sim\left(y_{0}, y_{1}, \ldots, y_{n-2}\right)=\left(z_{0}, z_{1}, \ldots, z_{n-2}\right)$, where $z_{i}=\sim y_{n-2-i}$ for each $i=0,1, \ldots, n-2$.

\section{Kleene algebras}

If $P$ is a prime ideal of a De Morgan algebra $M$, let $R(P)$ be the largest lattice-congruence having $P$ as a congruence-class. Of course, the partition of $M$ associated with $R(P)$ is $\{P, M \backslash P\}$. The partition associated with the lattice-congruence $R(P) \cap R(g(P))$ is $\{M \backslash(P \cup g(P)), P \backslash g(P), g(P) \backslash P, P \cap g(P)\}$, though, of course, we are not implying that these four congruence-classes are necessarily distinct. If $x \in M \backslash(P \cup g(P))$ then $x \in P \cap g(P) ;$ if $x \in P \cap g(P)$ then $\sim x \in M \backslash(P \cup g(P))$; if $x \in P \backslash g(P)$ then $\sim x$ is also in $P \backslash g(P)$; if $x \in g(P) \backslash P$ then $\sim x$ is also in $g(P) \backslash P$. Hence, $R(P) \cap R(g(P))$ is a mongruence and $M /(R(P) \cap R(g(P)))$ is isomorphic to 2 if and only if $P=g(P), M /(R(P) \cap R(g(P)))$ is isomorphic to 3 if and only if $P$ and $g(P)$ are comparable but not equal, and $M /(R(P) \cap R(g(P)))$ is isomorphic to $T=\{0, a, b, 1: 0=a \wedge b<a, b<a \vee b=1, \sim 0=1, \sim a=a, \sim b=b\}$ 
if and only if all four congruence-classes are distinct. In order to describe the dual of $K$ we need a consequence of the following result; we will need the full generality of the result later in this section.

THEOREM 3.1. Let $\Phi$ be a r congruence on a De Morgan algebra $M$ and let $\theta: M \rightarrow M / \Phi$ be the canonical epimorphism. Then

$$
\Phi=n\left\{R\left(\theta^{-1}(P)\right) \cap R\left(g\left(\theta^{-1}(P)\right)\right): P \in \operatorname{Pr}(M / \Phi)\right\} \text {. }
$$

Proof. Let $x, y \in M$ be such that $x \equiv y(\Phi)$. Then $\theta(x)=\theta(y)$. of course, $\theta^{-1}(P) \in \operatorname{Pr}(M)$ and, as $\theta$ is a r-homomorphism, $g\left(\theta^{-1}(P)\right)=M \backslash \theta^{-1}(P)=\theta^{-1}(M / \Phi \backslash P)=\theta^{-1}(g(P))$ for each $P \in \operatorname{Pr}(M / \Phi)$. As $x \in \theta^{-1}(P)$ if and only if $y \in \theta^{-1}(P)$ and $x \in \theta^{-1}(g(P))$ if and only if $y \in \theta^{-I}(g(P))$ for each $P \in \operatorname{Pr}(M / \Phi)$, we have

$$
x \equiv y \cap\left\{R\left(\theta^{-1}(P)\right) \cap R\left(g\left(\theta^{-1}(P)\right)\right): P \in \operatorname{Pr}(M / \Phi)\right\} \text {. }
$$

Conversely, suppose $a, b \in M$ and yet $a \neq b(\Phi)$. Then $\theta(a) \neq \theta(b)$ and so there exists $P \in \operatorname{Pr}(M / \Phi)$ such that $\theta(a) \in P$ and $\theta(b) \notin P$, or vice-versa. Then $a \neq b\left(R\left(\theta^{-1}(P)\right)\right)$ and $a \neq b\left(R\left(\theta^{-1}(P)\right) \cap R\left(g\left(\theta^{-1}(P)\right)\right)\right)$, and the result follows.

Let $\Phi$ be $\omega$, the smallest congruence on $M$, whereby $x \equiv y(\omega)$ $(x, y \in M)$ if and only if $x=y$. Then $M / \Phi=M$ and so Theorem 3.1 implies that

$$
\cap\{R(P) \cap R(g(P)): P \in \operatorname{Pr}(M)\}=\omega .
$$

Hence we obtain the well known result that any De Morgan algebra is isomorphic to a subdirect product of copies of $T$ and its subalgebras 3 and 2 . Of course, $T, 3$, and 2 are the only subdirectly irreducible (simple) De Morgan algebras. All this was first established by BiaXynickiBirula [2, Theorem 2.1] and Kalman [11, Lemma 2], using other techniques. Since 2 and 3 are Kleene algebras while $T$ is not a Kleene algebra, we see that a De Morgan algebra is a Kleene algebra if and only if it is isomorphic to a subdirect product of copies of 2 and 3 . In other words, we have

THEOREM 3.2. The dual of $\mathrm{K}$ is isomorphic to the subcategory of g-Tode whose objects $(X, g)$ are such that $x$ and $g(x)$ are comparable for each $x \in X$. 
LEMMA 3.3. Each of the coproducts $3 \perp_{M} \perp^{3}, 3 \perp_{M} \perp_{M}$, and $4 \perp_{M} \perp^{4}$ is not a Kleene algebra.

Proof. The lemma can be proved by obtaining the required coproducts via Corollary 2.8; $\left.3 \perp_{M}\right\rfloor^{3}$ has a six-element planar graph, $3 \bigsqcup_{M}{ }_{M}$ has a ten element planar graph, while $4 \perp_{M} 4$ has a twenty element non-planar graph. However, it is a simple matter to establish the lemma by making use of Theorems 2.4 and 3.2; $(\operatorname{Pr}(3), g)$ is the discrete space $\left\{x_{0}, x_{1}: x_{0}>x_{1}, g\left(x_{0}\right)=x_{1}, g\left(x_{1}\right)=x_{0}\right\},(\operatorname{Pr}(4), g)$ is the discrete space $\left\{y_{0}, y_{1}, y_{2}: y_{0}>y_{1}>y_{2}, g\left(y_{0}\right)=y_{2}, g\left(y_{1}\right)=y_{1}, g\left(y_{2}\right)=y_{0}\right\}$. In $(\operatorname{Pr}(3), g) \times(\operatorname{Pr}(3), g), g\left(\left(x_{0}, x_{1}\right)\right)=\left(g\left(x_{0}\right), g\left(x_{1}\right)\right)=\left(x_{1}, x_{0}\right)$, which is incomparable with $\left(x_{0}, x_{1}\right)$. Similarly $g\left(\left(y_{0}, y_{2}\right)\right)=\left(y_{2}, y_{0}\right)$ in $(\operatorname{Pr}(4), g) \times(\operatorname{Pr}(4), g)$ and $\left(y_{2}, y_{0}\right)$ is incomparable with $\left(y_{0}, y_{2}\right)$. Similarly $g\left(\left(x_{0}, y_{2}\right)\right)=\left(x_{1}, y_{0}\right)$ in $(\operatorname{Pr}(3), g) \times(\operatorname{Pr}(4), g)$ and $\left(x_{0}, y_{2}\right)$ is incomparable with $\left(x_{1}, y_{0}\right)$. The proof is now complete.

LEMMA 3.4. Let $B$ be a boolean algebra and $K$ be a Kleene algebra. Then $B \perp \perp_{M} K$ is a Kleene algebra.

Proof. Since all the prime ideals are maximal in a boolean algebra $B,(\operatorname{Pr} B, g)$ is an ordered space in which the partial order is equality and the involution $g$ is the identity function. Thus, if $h . k \in C_{m}(\operatorname{Pr}(B), K)$ and $x \in \operatorname{Pr}(B)$,

$$
\begin{aligned}
(h \wedge \sim h)(x)=h(x) \wedge(\sim h)(x)=h(x) & \wedge \sim h(g(x))=h(x) \wedge \\
& \sim(h(x)) \leq k(x) \vee \sim k(x)=(k \vee \sim k)(x) .
\end{aligned}
$$

Thus the lemma follows from Theorem 2.7.

THEOREM 3.5. Let $\left\{K_{i}\right\}$ be a set of Kleene algebras. Then $\perp_{M}{ }_{i}$ is a Kleene algebra if and only if at most one of the $K_{i}$ is not a boolean algebra.

Proof. Due to Corollary 2.6, Lemma 3.4, and the "associativity" of coproducts, $\left.\perp\right|_{M}{ }_{i}$ is a Kleene algebra when all but possibly one of the $K_{i}$ is not boolean. 
On the other hand, suppose the coproduct in $M$ of the $K_{i}$ is a Kleene algebra and yet there exist two, namely $K_{i_{1}}$ and $K_{i_{2}}$, which are not boolean. Then $K_{i_{1}}$ and $K_{i_{2}}$ each have either 3 or 4 as a subalgebra and so one of $3 \perp_{M} \perp^{3}, 3 \perp_{M} \perp_{M}$ or $4 \perp_{M} \perp^{4}$ is isomorphic to a subalgebra of $\perp_{M} K_{i}$, due to Corollary 2.5 and its proof. Then Lemma 3.4 supplies the required contradiction.

Because a kleene algebra is isomorphic to a subdirect product of copies of 3 and its subalgebra 2 , there is always a homomorphism of a given Kleene algebra into 3 which distinguishes distinct elements of the algebra. Hence, due to Grätzer [9, Exercise 9, p. 138], the category $k$ has arbitrary coproducts. In Theorem 3.8 below, we exhibit a property of such coproducts; we present this after developing two results, which are of independent interest.

Cignoli [4, Corollaries 3.3, 4.4] showed that the categories $M$ and $K$ are injectively complete. From general theory [13, Proposition 2.1 and Theorem 2.3], a variety of algebras is injectively complete if and only if it is residually small and has both the congruence extension property and the amalgamation property. Thus $M$ and $K$ must have these three properties. Since the only subdirectly irreducible members of $M$ are the four element algebra $T$ together with its subalgebras 3 and $2, M$ and $K$ are residually small (cf. Taylor [13, Theorem 1.2 (ii)]); the aim of the next two results is to provide alternative proofs of the congruence extension and amalgamation properties for $M$ and $K$.

THEOREM 3.6. M, and so $K$, has the congruence extension property. Proof. Let $M$ be a subalgebra of De Morgan algebra $M_{1}$ and let $\Phi$ be a congruence on $M$. Due to Theorem 3.1,

$$
\Phi=\cap\left\{R\left(\theta^{-1}(P)\right) \cap R\left(g\left(\theta^{-1}(P)\right)\right): P \in \operatorname{Pr}(M / \Phi)\right\},
$$

where $\theta: M \rightarrow M / \Phi$ is the canonical epimorphism. Now for each prime ideal $P \in \operatorname{Pr}(M / \Phi), \theta^{-1}(P)$ is a prime ideal of the sublattice $M$ of the distributive lattice $M_{1}$ and so, by [1, Proof of Theorem 5, p. 74] (see also [9, Exercise 3, p. 100]), there is at least one prime ideal $P_{1}$ of 
$M_{1}$ such that $P_{1} \cap M=\theta^{-1}(P)$ and $P_{1} \cap\left(M \backslash \theta^{-1}(P)\right)=\varnothing$. Since $M$ is a $\sim$-subalgebra of $M$, we must also have $\sim \theta^{-1}(P)=M \cap \sim P_{1}$ and $\sim P_{1} \cap\left(M \vee \theta^{-1}(P)\right)=\emptyset$, so that $g\left(\theta^{-1}(P)\right)=M \cap g\left(P_{1}\right)$ and $g\left(P_{1}\right) \cap\left(M \backslash g\left(\theta^{-1}(P)\right)\right)=\emptyset$. For each $P \in \operatorname{Pr}(M / \Phi)$, choose a prime ideal $P_{1} \in \operatorname{Pr}\left(M_{1}\right)$ with the above properties. Define

$$
\Phi_{1}=\cap\left\{R\left(P_{1}\right) \cap R\left(g\left(P_{1}\right)\right): P_{1} \text { is related to } P \in \operatorname{Pr}(M / \Phi) \text {, as above }\right\} \text {. }
$$

Then $\Phi_{1}$ is a congruence on $M_{1}$ and a routine check shows that $\Phi_{1} \cap(M \times M)=\Phi$, as required.

Since $M$ and $K$ are each varieties with the congruence extension property and such that each subalgebra of a subdirectly irreducible algebra is itself subdirectly irreducible, we can show that each of $M$ and $K$ has the amalgamation property if we prove that each amalgam

$$
(A ; B, \gamma, C, \delta ; \gamma: A \rightarrow B \text { and } \delta: B \rightarrow C \text { are monomorphisms), }
$$

where $A, B$, and $C$ are subdirectly irreducible algebras in $M$ or $\mathrm{K}$, can be amalgamated. This is due to a deep result of Grätzer and Lakser [10, Theorem 3]. The verification that such an amalgam can here be amalgamated is a simple matter and so we have

THEOREM 3.7. Both $M$ and $K$ have the amalgamation property.

Because of Theorems 3.6 and 3.7 and another result of Grätzer and Lakser [10, Theorem 4] we have

THEOREM 3.8. Let $A$ and $B$ be Kleene algebras, and $A_{1}$ and $B_{1}$ be subalgebras of $A$ and $B$, respectively. Then the subalgebra of $A \perp \perp_{K}^{B}$ generated by $A_{1}$ and $B_{1}$ is isomorphic to $A_{1} \perp_{K}{ }_{K}$.

\section{References}

[1] Raymond Balbes and Philip Dwinger, Distributive Zattices (University of Missouri Press, Columbia, Missouri, 1974). 
[2] A. Bialynicki-Birula, "Remarks on quasi-boolean algebras", BuZZ. Acad. Polon. Sci. Cl. III 5 (1957), 615-619.

[3] A. Białynicki-Birula and H. Rasiowa, "On the representation of quasiboolean algebras", Bulz. Acad. Polon. Sci. Cl. III 5 (1957), 259-261.

[4] Roberto Cignoli, "Injective De Morgan and Kleene algebras", Proc. Amer. Math. Soc. 47 (1975), 269-278.

[5] William H. Cornish, "Compactness of the clopen topology and applications to ideal theory", General Topology and Appl. 5 (1975), 347-359.

[6] William H. Cornish, "On H. Priestley's dual of the category of bounded distributive lattices", Mat. Vesnik 12 (27) (1975), $329-332$.

[7] William H. Cornish, "Ordered topological spaces and the coproduct of bounded distributive lattices", Colloq. Math. (to appear).

[8] Brian A. Davey, "Free products of bounded distributive lattices", Algebra Universalis 4 (1974), 106-107.

[9] George Grätzer, Lattice theory. First concepts and distributive Zattices (Freeman, San Francisco, 1971).

[10] G. Grätzer and H. Lakser, "The structure of pseudocomplemented distributive lattices. II: Congruence extension and amalgamation", Trons. Amer. Math. Soc. 156 (1971), 343-358.

[11] J.A. Kalman, "Lattices with involution", Trans. Amer. Math. Soc. 87 (1958), 485-491.

[12] Iona Petrescu, "Injective objects in the category of Morgan algebras", Rev. Roumonie Math. Pures. Appl. 16 (1971), 921-926.

[13] Walter Taylor, "Residually small varieties", Algebra Universalis 2 (1972), 33-53.

School of Mathematical Sciences,

Flinders University of South Australia,

Bedford Park, South Australia. 